transmigration of RASF and the following invasion of cartilage are unknown.

Methods Transmigration assays of RASF through human umbilical vein endothelial cell (HUVECs) layers cultured in transwell chambers were performed. The number of migrating RASFs was evaluated at different time points (4-16h). We also used the SCID mouse model of RA: $1.5 \times 10^{5}$ RASF were implanted subcutaneously in a gelatin matrix containing human healthy cartilage at the ipsilateral site (I) and a gelatin matrix with cartilage without RASF at the contralateral site (C). The neoangiogenesis into the implants and the invasion of RASF into the cartilage was analysed after $35 \mathrm{~h}$ and $6,12,18$, 24 and 30 days.

Results Transmigration ofRASF through the endothelialmonolayer was detectable after $4 \mathrm{~h}(21.8 \pm 0.2 \%)$. Neoangiogenesis and vessel formation into both implants could be observed already after $35 \mathrm{~h}$, being more prominent at the ispilateral site. The invasion started at the ipsilateral site at day 18 (score 0.86), at the contralateral site at day 24 after implantation (score 0.43 ) and increased continuously over time. RASF could be detected immunohistochemically in the blood and in all spleens of the SCID mice at every time point.

Conclusion RASF are able to migrate through endothelial cell layers. An increased neoangiogenesis at the ipsilateral site containing RASF is most likely mediated by the activated RASF. The transmigration assays showed that RASF are able to transmigrate through vessel walls. Neoangiogenesis, transmigration and invasion therefore appear to be very early events in RA and play an important role in RASF-mediated cartilage destruction.

\title{
A145 MIGRATORY POTENTIAL AND INFLUENCE ON NEOANGIOGENESIS OF RHEUMATOID ARTHRITIS SYNOVIAL FIBROBLASTS
}

B Zimmermann, ${ }^{1}$ S Lefèvre, ${ }^{1}$ S Fischer, ${ }^{2}$ J Gansler, ${ }^{2}$ A Lehr, ${ }^{3}$ S Rehart, ${ }^{3}$ H Stürz, ${ }^{4} \mathrm{~J}$ Steinmeyer, ${ }^{5}$ U Müller-Ladner, ${ }^{1}$ E Neumann ${ }^{1}{ }^{1}$ Internal Medicine and Rheumatology, Justus-Liebig-University Gießen, Kerckhoff-Klinik Bad Nauheim, Germany; ${ }^{2}$ Department of Biochemistry, Justus-Liebig-University Gießen, Germany; ${ }^{3}$ Department of Orthopedics, Markuskrankenhaus Frankfurt, Germany; ${ }^{4}$ Department of Orthopedics and Orthopedic Surgery, Justus-Liebig-University Gießen, Germany; ${ }^{5}$ Department of Orthopedics and Exp Orthopedics, Justus-Liebig-University Gießen, Germany

10.1136/ard.2010.129643v

Background Rheumatoid arthritis (RA) is a chronic inflammatory joint disease including joint destruction that is characterised by starting in single joints and then spreading to non-affected joints. We recently demonstrated the long-distance migratory behavior of RA synovial fibroblasts (SF) via the blood stream and the invasion of distant cartilage in the SCID mouse model of RA. The mechanisms of the endothelial 